\title{
Risco de ortorexia nervosa e o comportamento alimentar de estudantes de nutrição
}

Risk of orthorexia nervous and the food behavior of nutrition students

\author{
B. C. Rodrigues ${ }^{1}$, G. N. S. de Oliveira ${ }^{1}$, E. I. G. e Silva ${ }^{1}$, C. M. B. de O. \\ Messias $^{1,2 *}$ \\ ${ }^{1}$ Colegiado de nutrição, Universidade de Pernambuco Campus Petrolina, 56.328-903, Petrolina-PE, Brasil \\ ${ }_{2}^{2}$ Programa de Pós-graduação Formação de Professores e Práticas Interdisciplinares (PPGFPPI), Universidade de \\ Pernambuco Campus Petrolina, 56.328-903, Petrolina-PE, Brasil \\ *cristhiane.omena@upe.br
}

(Recebido em 22 de abril de 2017; aceito em 18 de julho de 2017)

\begin{abstract}
Ortorexia Nervosa é um desvio alimentar que envolve obsessividade patológica por alimentação saudável. O objetivo do presente estudo foi avaliar o comportamento de risco para desenvolvimento de ortorexia nervosa e o comportamento alimentar de estudantes de bacharelado em Nutrição. Trata-se de uma pesquisa de campo quantitativa, tipo descritivo e transversal, desenvolvida entre agosto de 2015 e julho de 2016, com discentes do curso de Nutrição. Os instrumentos utilizados foram o Orto-15, para avaliar o comportamento de risco para desenvolver ortorexia nervosa, e o questionário de frequência alimentar, para investigação do consumo alimentar. Grande parte dos discentes apresentaram traços de ortorexia nervosa, sendo o sexo masculino com maior prevalência. Não houve associação estatística significativa entre os grupos dos diferentes semestres analisados, nem quanto ao sexo. Também foi observada inadequação de hábitos alimentares. Faz-se necessário, portanto, uma conscientização e abordagem acerca do tema, em especial aos que lidam com a promoção da saúde, por atuarem diretamente com os possíveis grupos de risco.
\end{abstract}

Palavras-chave: Hábitos Alimentares; Alimentos; Dieta; Nutrição.

Orthorexia Nervosa is a food diversion that involves pathological obsessiveness for healthy eating. The objective of the present study was to evaluate the risk behavior for the development of orthorexia nervosa and the eating behavior of baccalaureate Nutrition students. It is a quantitative field research, descriptive and transversal, developed between August 2015 and July 2016, with Nutrition students. The instruments used were Orto-15, to evaluate the risk behavior to develop orthorexia nervosa, and the food frequency questionnaire, to investigate food consumption. Most of the students presented traces of orthorexia nervosa, being the masculine sex with greater prevalence. There was no statistically significant association between the groups of the semesters analyzed, nor with regard to sex. Also, inadequate eating habits were observed. It is necessary to raise awareness and approach on the subject, especially those that deal with health promotion, by acting directly with the possible risk groups.

Keywords: Food Habits; Food; Diet; Nutrition.

\section{INTRODUÇÃO}

Dentre as ações humanas consideradas indispensáveis, a alimentação surge não só por razões biológicas evidentes, mas também por abranger aspectos econômicos, sociais, científicos, políticos, psicológicos e culturais imprescindíveis para a progressão das sociedades [1].

Na contemporaneidade, os hábitos alimentares se remodelam de acordo com o estilo de vida presenciado. Tal estilo, marcado pelo ritmo frenético e consequentemente escassez de tempo, vem caracterizando o imediatismo de todas as atividades, incluindo a alimentação. O que resulta em escolhas por alimentos do tipo "fast-food", sendo visto como útil àqueles que agregam tempo e trabalho [2].

Somado a esse quadro, a mídia faz um "bombardeio" incessante do culto ao corpo, padrões de beleza e estética, atividades físicas e alimentação, que muitas vezes não condizem com a 
realidade, interferindo na vida dos indivíduos acometidos por transtornos alimentares, caracterizados por um padrão comportamental de compulsões [3].

Os transtornos de conduta alimentar formam um grande grupo de distúrbios, dentre os mais conhecidos e prevalentes estão a anorexia nervosa (AN) e a bulimia nervosa (BN) [4]. A ortorexia nervosa $(\mathrm{ON})$, quando comparada a esses transtornos, apresenta comportamentos similares e distintos com os demais [5].

Não aludida pela Quinta Edição do Manual de Diagnóstico e Estatístico dos Transtornos Mentais (DSM-V) e pela Décima Revisão da Classificação Internacional de Doenças (CID-10) da Organização Mundial de Saúde, a ON é descrita como um desvio de conduta alimentar obsessivo-patológico relacionada à alimentação "saudável", qualidade dos alimentos e pureza da dieta $[6,7]$.

Conectados a uma sensação ilusória de segurança e desejo ao exercer um controle total sobre a própria vida, os indivíduos com transtornos alimentares seguem um padrão de dietas descontínuas, insuficientes e desequilibradas [8], que por vezes resultam em carências nutricionais devido à exclusão de vários grupos alimentares [6,9].

A alimentação saudável é um dos mais importantes conceitos enfatizados quando se analisa questões relacionadas com a melhoria da saúde. Estudantes e profissionais da área são cobrados constantemente por um padrão saudável e estético, justificando sua vulnerabilidade a transtornos alimentares [10]. Com base no exposto, o objetivo do presente estudo foi avaliar o comportamento de risco para desenvolvimento de ortorexia nervosa em estudantes de bacharelado em Nutrição no município de Petrolina - PE.

\section{MATERIAL E MÉTODOS}

Trata-se de uma pesquisa de campo quantitativo, do tipo descritivo e transversal, desenvolvido no período de agosto de 2015 a julho de 2016, com discentes do curso de Nutrição de uma instituição pública, com idade a partir de 18 anos, de ambos os sexos. A pesquisa foi submetida e aprovada pelo comitê de ética da Universidade de Pernambuco - UPE, CAAE: 43754215.5.0000.5207, $\mathrm{n}^{\circ}$ do parecer: 1.050.149, em Maio de 2015.

O instrumento de pesquisa utilizado foi o Orto-15, para a avaliação do comportamento de risco para desenvolver a ortorexia nervosa. Os resultados do Orto-15 foram contabilizados e classificados a partir do quadro elaborado por Donini et al. (2005) [11].

Para a investigação do consumo alimentar foi elaborado e utilizado um questionário de frequência alimentar (QFA) que contém uma listagem de alimentos estruturados em três grupos distintos: Alimentos construtores (leite e produtos lácteos, carnes, ovos, miúdos/vísceras e leguminosas); Alimentos reguladores (hortaliças e frutas) e alimentos energéticos (cereais, raízes, tubérculos, pães, massas, bolos, gorduras, açúcares, doces e bebidas açucaradas). Em cada um dos grupos de alimentos foi determinado o consumo alimentar de duas maneiras: uma classificando-se em frequência semanal e outra o consumo diário. O questionário foi estruturado com 58 opções alimentares. Além disso, foi composto por 3 opções de bebidas e infusões. Para verificar a frequência de consumo utilizou-se: nunca ou menos de uma vez ao mês; uma a três vezes ao mês; uma vez por semana; duas a quatro vezes por semana; cinco a seis vezes por semana; uma vez ao dia; duas a três vezes ao dia; quatro a cinco vezes ao dia; seis ou mais vezes ao dia, além da opção sazonal.

Todos os dados foram compilados no Microsoft Excel (2010). Após a compilação, efetuou-se análise estatística descritiva: média, desvio padrão e porcentagens. Para avaliar a associação existente entre as variáveis categóricas ortorexia e sexo aplicou-se o teste exato de Fisher. Para as variáveis ortorexia e escolaridade, o teste $G$-Independência. Consideraram-se significativos os testes que apresentarem $p<0,05$. 


\section{RESULTADOS E DISCUSSÃO}

Foram inclusos 113 discentes, com a representatividade de 51\% dos matriculados no curso de Nutrição da Universidade em 2015, sendo 84\% (n=95) do sexo feminino e 16\% ( $n=18)$ do sexo masculino.

Constatou-se que 94,69\% dos discentes apresentaram traços de ortorexia, enquanto os demais não apresentaram risco. Quando os dados sobre o comportamento ortoréxico foram relacionados ao período cursado, verificou-se que todos apresentaram índices elevados de traços de ortorexia nervosa (Tabela 1).

Tabela 1. Distribuição dos discentes do curso de nutrição segundo o comportamento ortoréxico e a série cursada, Petrolina-PE, 2016.

\begin{tabular}{|c|c|c|c|c|c|c|}
\hline \multirow{3}{*}{$\begin{array}{l}\text { Comportamento } \\
\text { Ortoréxico }\end{array}$} & \multicolumn{5}{|c|}{ Período } & \multirow{3}{*}{ Valor de $p^{*}$} \\
\hline & $1^{\circ}$ & $3^{\circ}$ & $5^{\circ}$ & $8^{\circ}$ & Total & \\
\hline & $\mathrm{N}(\%)$ & $\mathrm{N}(\%)$ & $\mathrm{N}(\%)$ & $\mathrm{N}(\%)$ & $\mathrm{N}(\%)$ & \\
\hline Sim & $29(96,7)$ & $24(92,3)$ & $34(97,1)$ & $20(90,9)$ & $107(94,7)$ & \multirow{3}{*}{$p=0,6715$} \\
\hline Não & $1(3,3)$ & $2(7,7)$ & $1(2,9)$ & $2(9,1)$ & $6(5,3)$ & \\
\hline Total por período & $30(100)$ & $26(100)$ & $35(100)$ & $22(100)$ & $113(100)$ & \\
\hline
\end{tabular}

* Teste $\mathrm{G}$ - Independência.

Dados apresentados como número (\%)

Referente ao sexo, $100 \%$ dos estudantes do sexo masculino e $93,68 \%$ do sexo feminino apresentaram traços de ortorexia. Não houve associação estatística significativa entre as variáveis ortorexia e período cursado ( $p=0,6715)$, e ortorexia e sexo $(p=0,5872)$.

$\mathrm{Na}$ análise do questionário de frequência alimentar por grupo alimentar, dentre os alimentos de fonte energética (Tabela 2), o açúcar apresentou maior frequência de consumo, sendo utilizado de duas a três vezes ao dia por mais de um terço dos estudantes. $\mathrm{O}$ arroz cozido foi consumido uma vez ao dia, o cuscuz e o macarrão destacaram-se no consumo de duas à quatro vezes por semana.

Tabela 2. Frequência do consumo de alimentos do grupo dos alimentos energéticos por discentes do curso de Nutrição, Petrolina-PE, 2016.

\begin{tabular}{|c|c|c|c|c|c|c|}
\hline \multicolumn{7}{|c|}{ Consumo } \\
\hline & \multicolumn{3}{|c|}{ Vezes por semana } & \multicolumn{3}{|c|}{ Vezes por dia } \\
\hline & 1 & $2-4$ & $5-6$ & 1 & $2-3$ & 4-5 \\
\hline Açúcar & $\begin{array}{l}7,07 \% \\
(\mathrm{n}=8)\end{array}$ & $\begin{array}{l}7,96 \% \\
(\mathrm{n}=9)\end{array}$ & $\begin{array}{l}9,73 \% \\
(n=11)\end{array}$ & $\begin{array}{l}18,58 \% \\
(n=21)\end{array}$ & $\begin{array}{l}41,59 \% \\
(n=47)\end{array}$ & $\begin{array}{l}3,53 \% \\
(n=4)\end{array}$ \\
\hline Arroz & $\begin{array}{l}4,42 \% \\
(n=5)\end{array}$ & $\begin{array}{l}7,07 \% \\
(\mathrm{n}=8)\end{array}$ & $\begin{array}{l}22,12 \% \\
(n=25)\end{array}$ & $\begin{array}{l}41,59 \% \\
(n=47)\end{array}$ & $\begin{array}{l}18,58 \% \\
(n=21)\end{array}$ & $\begin{array}{l}2,65 \% \\
(n=3)\end{array}$ \\
\hline Cuscuz & $\begin{array}{l}23,89 \% \\
(n=27)\end{array}$ & $\begin{array}{l}40,70 \% \\
(n=46)\end{array}$ & $\begin{array}{l}7,07 \% \\
(\mathrm{n}=8)\end{array}$ & $\begin{array}{l}7,96 \% \\
(n=9)\end{array}$ & $\begin{array}{l}7,07 \% \\
(\mathrm{n}=8)\end{array}$ & 0 \\
\hline Macarrão & $\begin{array}{l}22,12 \% \\
(n=25)\end{array}$ & $\begin{array}{l}37,16 \% \\
(n=42)\end{array}$ & $\begin{array}{l}7,07 \% \\
(\mathrm{n}=8)\end{array}$ & $\begin{array}{l}7,07 \% \\
(\mathrm{n}=8)\end{array}$ & 0 & $\begin{array}{l}0,88 \% \\
(n=1)\end{array}$ \\
\hline
\end{tabular}

Observou-se em relação ao grupo de alimentos construtores (Tabela 3) que os mais frequentes foram: feijão, frango, carne bovina assada e ovo. 
Tabela 3. Frequência do consumo de alimentos do grupo dos construtores por discentes do curso de Nutrição, Petrolina-PE, 2016.

\begin{tabular}{|c|c|c|c|c|c|c|}
\hline \multicolumn{7}{|c|}{ Consumo } \\
\hline & \multicolumn{2}{|c|}{ Por semana } & \multicolumn{4}{|c|}{ Por dia } \\
\hline & 1 & $2-4$ & $5-6$ & 1 & $2-3$ & $4-5$ \\
\hline Feijão & $\begin{array}{l}2,65 \% \\
(\mathrm{n}=3)\end{array}$ & $\begin{array}{l}17,69 \% \\
(n=20)\end{array}$ & $\begin{array}{c}23 \% \\
(n=26)\end{array}$ & $\begin{array}{l}35,39 \% \\
(n=40)\end{array}$ & $\begin{array}{l}9,73 \% \\
(n=11)\end{array}$ & $\begin{array}{l}1,76 \% \\
(\mathrm{n}=2)\end{array}$ \\
\hline $\begin{array}{l}\text { Carne* } \\
\text { assada }\end{array}$ & $\begin{array}{l}18,58 \% \\
(n=21)\end{array}$ & $\begin{array}{l}45,13 \% \\
(n=51)\end{array}$ & $\begin{array}{l}6,19 \% \\
(n=7)\end{array}$ & $\begin{array}{l}3,53 \% \\
(n=4)\end{array}$ & $\begin{array}{l}5,30 \% \\
(n=6)\end{array}$ & 0 \\
\hline $\begin{array}{l}\text { Frango } \\
\text { assado }\end{array}$ & $\begin{array}{l}14,15 \% \\
(n=16)\end{array}$ & $\begin{array}{l}46,01 \% \\
(n=52)\end{array}$ & $\begin{array}{l}14,15 \% \\
(n=16)\end{array}$ & $\begin{array}{l}7,96 \% \\
(\mathrm{n}=9)\end{array}$ & $\begin{array}{l}7,07 \% \\
(\mathrm{n}=8)\end{array}$ & $\begin{array}{l}0,88 \% \\
(\mathrm{n}=1)\end{array}$ \\
\hline Ovo & $\begin{array}{l}23,89 \% \\
(n=27)\end{array}$ & $\begin{array}{l}39,82 \% \\
(n=45)\end{array}$ & $\begin{array}{l}7,07 \% \\
(\mathrm{n}=8)\end{array}$ & $\begin{array}{c}8,84 \% \\
(\mathrm{n}=10)\end{array}$ & $\begin{array}{l}4,42 \% \\
(n=5)\end{array}$ & $\begin{array}{l}2,65 \% \\
(\mathrm{n}=3)\end{array}$ \\
\hline $\begin{array}{l}\text { Leite } \\
\text { integral }\end{array}$ & $\begin{array}{c}8,84 \% \\
(n=10)\end{array}$ & $\begin{array}{l}13,27 \% \\
(n=15)\end{array}$ & $\begin{array}{l}6,19 \% \\
(n=7)\end{array}$ & $\begin{array}{l}13,27 \% \\
(n=15)\end{array}$ & $\begin{array}{l}12,38 \% \\
(n=14)\end{array}$ & $\begin{array}{l}2,65 \% \\
(\mathrm{n}=3)\end{array}$ \\
\hline Iogurte & $\begin{array}{l}18,58 \% \\
(\mathrm{n}=21)\end{array}$ & $\begin{array}{l}26,54 \% \\
(n=30)\end{array}$ & $\begin{array}{l}5,30 \% \\
(n=6)\end{array}$ & $\begin{array}{l}6,19 \% \\
(n=7)\end{array}$ & $\begin{array}{l}1,76 \% \\
(n=2)\end{array}$ & 0 \\
\hline
\end{tabular}

*Carne bovina.

A partir desses dados, evidenciou-se que o consumo mais relevante dos alimentos construtores foi o do feijão, onde a maior parte da amostra $(n=53)$ realiza o consumo diário, dentre estes, a maior representatividade cita consumir uma vez ao dia. A maioria dos estudantes relatou consumir carnes e ovos semanalmente, sendo o consumo de duas a quatro vezes por semana para ambos. Observou-se também, que o leite o mais consumido foi o integral, sendo consumido diariamente por $29,20 \%(n=33)$, e semanalmente por $28,31 \%(n=32)$ do total da amostra. O iogurte aparece com maior frequência de consumo semanal, estando presente em $50,44 \%(n=57)$ das dietas dos estudantes, e diariamente por apenas 7,96\% $(n=9)$.

Quanto ao consumo de alimentos reguladores, as hortaliças mais consumidas foram a alface e o tomate (frequência de consumo de duas a quatro vezes por semana). Em relação ao consumo habitual de frutas, as que apresentaram maior frequência de consumo foram a banana, manga e melancia, ambas de duas a quatro vezes na semana (Tabela 4).

O presente estudo verificou que $94,69 \%$ dos discentes do curso de Nutrição apresentavam comportamento de risco para ortorexia nervosa. Ao buscar dados na literatura, é evidenciado por Pontes (2012) [12] a prevalência de 83\% para a ortorexia nervosa em alunos do curso de Técnicos em Nutrição e Dietética. No mesmo ano, Nassau (2012) [14] avaliando estudantes do curso de Nutrição do $1^{\circ}$ ao $8^{\circ}$ semestre, verificou a incidência em $27,4 \%$ da amostra para traços ortoréxicos, sendo significativo principalmente para $2^{\circ}$ semestre (43,5\%), enquanto $69,4 \%$ estavam em risco para esse comportamento, com $90 \%$ de risco para os que cursavam o $3^{\circ}$ e $8^{\circ}$ semestre, e 3,2\% não apresentaram risco algum de ortorexia.

O estudo desenvolvido por Souza \& Rodrigues (2014) [4], avaliando estudantes de Nutrição quanto à ortorexia nervosa, observou que $88,7 \%$ apresentavam risco de desenvolver comportamento ortoréxico. Este estudo, ao relacionar o comportamento ortoréxico com a série cursada mostrou que não houve associação entre as variáveis do comportamento ortoréxico e período cursado, fato também verificado em nosso estudo. 
Tabela 4. Frequência do consumo de alimentos do grupo dos reguladores por discentes do curso de Nutrição, Petrolina-PE, 2016.

\begin{tabular}{|c|c|c|c|c|c|c|}
\hline \multicolumn{7}{|c|}{ Consumo } \\
\hline \multicolumn{4}{|c|}{ Por semana } & \multicolumn{3}{|c|}{ Por dia } \\
\hline & 1 & $2-4$ & $5-6$ & 1 & $2-3$ & $4-5$ \\
\hline Alface & $\begin{array}{l}12,38 \% \\
(n=14)\end{array}$ & $\begin{array}{l}35,39 \% \\
(n=40)\end{array}$ & $\begin{array}{c}8,84 \% \\
(n=10)\end{array}$ & $\begin{array}{c}9,73 \% \\
(\mathrm{n}=11)\end{array}$ & $\begin{array}{l}1,76 \% \\
(n=2)\end{array}$ & 0 \\
\hline Tomate & $\begin{array}{l}7,07 \% \\
(\mathrm{n}=8)\end{array}$ & $\begin{array}{l}30,97 \% \\
(n=35)\end{array}$ & $\begin{array}{l}16,81 \% \\
(n=19)\end{array}$ & $\begin{array}{l}10,61 \% \\
(n=12)\end{array}$ & $\begin{array}{l}7,96 \% \\
(n=9)\end{array}$ & $\begin{array}{l}2,65 \% \\
(\mathrm{n}=3)\end{array}$ \\
\hline Banana & $\begin{array}{l}11,50 \% \\
(n=13)\end{array}$ & $\begin{array}{l}38,94 \% \\
(n=44)\end{array}$ & $\begin{array}{l}14,15 \% \\
(n=16)\end{array}$ & $\begin{array}{l}7,96 \% \\
(n=9)\end{array}$ & $\begin{array}{l}6,19 \% \\
(n=7)\end{array}$ & $\begin{array}{l}2,65 \% \\
(n=3)\end{array}$ \\
\hline Manga & $\begin{array}{l}17,69 \% \\
(n=20)\end{array}$ & $\begin{array}{l}12,40 \% \\
(n=14)\end{array}$ & $\begin{array}{l}3,53 \% \\
(n=4)\end{array}$ & $\begin{array}{l}1,76 \% \\
(\mathrm{n}=2)\end{array}$ & $\begin{array}{l}1,76 \% \\
(n=2)\end{array}$ & 0 \\
\hline Melancia & $\begin{array}{l}20,35 \% \\
(n=23)\end{array}$ & $\begin{array}{l}14,15 \% \\
(n=16)\end{array}$ & $\begin{array}{l}5,30 \% \\
(\mathrm{n}=6)\end{array}$ & 0 & $\begin{array}{l}0,88 \% \\
(\mathrm{n}=1)\end{array}$ & 0 \\
\hline
\end{tabular}

As desordens alimentares e distorções na imagem corporal parecem ser mais incidentes naqueles cuja profissão de forma direta ou indireta exijam um maior cuidado com a alimentação, estando mais sujeitos à pressão da sociedade quanto a manutenção do peso considerado adequado, que muitas vezes não condiz com o peso ideal, influenciando diretamente no desempenho da profissão [12]. São grupos vulneráveis à ortorexia estudantes da área da saúde, tais como estudantes de Nutrição, Educação Fisíca e Medicina, e profissionais da área da saúde, além das pessoas com maior visibilidade ao meio público $[6,10]$.

No estudo de Aksoydan \& Camci (2009) [13] sobre ortorexia nervosa, realizado com 94 artistas turcos, de ambos os gêneros, foi encontrada uma prevalência de $56,4 \%$, sendo a amostra constituída por cantores de ópera $(81,8 \%)$, bailarinos $(32,1 \%)$ e músicos da orquestra sinfônica $(36,4 \%)$. Os autores referem que para os turcos, os artistas são considerados modelos de aparência física e de estilo de vida, sendo essa a possível vulnerabilidade desse grupo.

A beleza física, a capacidade ou habilidade valorizada socialmente (como no esporte ou nas artes) valem como um capital físico que pode significar a distinção social e a possibilidade de ascensão ou manutenção do "status", como também pode ser convertido em dinheiro e poder econômico, afirma Pontes (2012) [12].

Observou-se, em nosso estudo, que $100 \%(n=18)$ dos estudantes do sexo masculino apresentaram traços de ortorexia. Esses resultados assemelham-se aos encontrados por Nassau [14], onde a prevalência de casos positivos para ortorexia foram 38,5\% para os homens contra $26,1 \%$ das mulheres. Em estudo com 878 estudantes de medicina turcos, de ambos os sexos (464 homens e 359 mulheres), a tendência foi significativamente mais alta entre estudantes do sexo masculino. Observou-se, ainda, maior ocorrência para ortorexia nervosa entre estudantes mais jovens e do sexo masculino [15].

Os achados do presente estudo devem ser interpretados com cautela, pois são diversos os construtos que envolvem a rotina dos discentes possibilitando o desenvolvimento de um comportamento ortoréxico. Tendo em vista isso, a aplicação do questionário de frequência alimentar é de grande importância, para que desta forma se conheça o real padrão de consumo alimentar da população analisada [16].

O consumo do grupo energético (Tabela 2) apontou ingestão repetitiva do açúcar de mesa, cuja tendência de consumo é motivo de preocupação, tendo em vista os efeitos indesejáveis na saúde provocados pelos açúcares adicionados na dieta, principalmente em grande quantidade. As consequências do consumo de "açúcares de adição" são várias, desde a corrosão do esmalte dentário resultante do metabolismo dos açúcares por bactérias, o comprometimento da auto regulação do balanço energético influenciando o mecanismo fome/saciedade, e o aumento da 
concentração de triglicerídeos e diminuição da concentração da lipoproteína de alta densidade [17]. Além disso, contribui para o ganho excessivo de peso e para o aparecimento de Doenças Crônicas não Transmissíveis, como hipertensão e diabetes.

Quanto ao consumo do grupo dos construtores (Tabela 3), evidenciou-se que o consumo de leguminosas é inadequado para a maior parte da população, 53,09\% $(n=60)$, sendo inferior a uma porção diária recomendada pelo Guia Alimentar para a População Brasileira [18]. Como consequência, uma parcela significativa da ingestão diária de macro e micronutrientes, em especial, proteínas, fibras, vitaminas do complexo B e minerais, como ferro, zinco e cálcio são privados da alimentação. Além disso, são alimentos com potencial de saciedade, pelo seu alto teor de fibras e de quantidade moderada de calorias por grama.

Resultados insatisfatórios também foram encontrados por Leite et al. (2011) [19], onde 87,5\% dos entrevistados consumiam feijão menos do que cinco dias na semana, bem como por Santos et al. [20], que em sua pesquisa verificou que apenas $28,33 \%$ faziam consumo diário e $52,5 \%$ semanal.

O consumo de carnes e ovos semanal vem a conferir, de modo geral, excelentes fontes de proteína e micronutrientes, como zinco e vitamina B12. Entretanto, as carnes vermelhas tendem a serem ricas em gorduras, principalmente gorduras saturadas, que, quando consumidas em excesso, aumentam o risco de doenças cardiovasculares e outras doenças crônicas. As carnes de aves também são ricas em proteínas de alto valor biológico, vitaminas e minerais, e podem apresentar elevado teor de gorduras saturadas, mas diferente das carnes vermelhas, a gordura está concentrada na pele. Os ovos são ricos em proteínas de alta qualidade, em minerais e em vitaminas, especialmente do complexo B [18].

Observou-se um baixo consumo de leite e derivados, esses resultados corroboram com os encontrados por Leite et al. (2011) [19], em que 69,1\% dos estudantes não faziam o consumo deste alimento. Estes alimentos são muito importantes por serem ricos em proteínas, em algumas vitaminas e, principalmente cálcio, e a depender do tipo pode ser rico em gorduras [18].

Quanto ao consumo de alimentos reguladores (Tabela 4), observou-se um inadequado consumo alimentar, pois de acordo com o Guia Alimentar para a População Brasileira [18], o consumo de frutas e hortaliças deve ser de pelo menos três porções diárias. Esses dados são preocupantes, pois esses grupos alimentares são ricos em fibra alimentar e diferentes tipos de vitaminas e minerais, que garantem a oferta de micronutrientes, oferecem uma quantidade pequena de calorias e fornecem antioxidantes.

Muniz et al. (2013) [21] constatou em sua pesquisa que atualmente apenas uma pequena parte da população atinge a recomendação diária de frutas e hortaliças, e que entre os jovens este consumo tendência a ser ainda menor. Relacionaram também que este consumo insuficiente é um dos principais fatores de risco para o aparecimento das Doenças Crônicas Não Transmissíveis.

Das bebidas e infusões questionadas, o café foi a de maior consumo pelos estudantes, de duas a quatro vezes por semana $(17,69 \%)$, enquanto $15,92 \%(n=18)$ e $15,04 \%(n=17)$, bebem de duas a três vezes e uma vez ao dia, respectivamente. Tais resultados corroboram com os obtidos por Bassols et al. (2008) [22], onde a maior parte dos estudantes $(74,4 \%)$ relataram fazer uso de substâncias estimulantes como o café. Diversas podem ser as razões para tal achado, como a ansiedade com as exigências do curso, o estresse [22], além de ser uma bebida que é consumida por uma parcela heterogênea da população por estar fortemente associada aos hábitos e costumes da sociedade brasileira [23].

\section{CONCLUSÃO}

Os achados do presente estudo revelam que a maior parte dos universitários apresentaram traços de ortorexia nervosa, sendo no sexo masculino a maior prevalência. Aliado a isto, os resultados referentes ao questionário de frequência alimentar evidenciam a inadequação de alguns hábitos alimentares, o que significa que apesar dos voluntários serem da área da saúde, e mais especificamente do curso de Nutrição, ainda existe uma dicotomia entre o saber teórico e a aplicação na prática diária. Convém mencionar que apesar da população possuir o traço de ortorexia nervosa não apresentam, ainda, as características do distúrbio mencionadas no presente 
trabalho. Prerrogativa importante no que se refere a intervenção e tratamento do possível transtorno.

Faz-se necessário, portanto, uma conscientização e abordagem acerca do tema, em especial aos que lidam com a promoção da saúde, por atuarem diretamente com os possíveis grupos de risco.

\section{AGRADECIMENTOS}

Ao Conselho Nacional de Desenvolvimento Científico e Tecnológico (CNPq) pela bolsa de iniciação científica concedida.

\section{REFERÊNCIAS BIBLIOGRÁFICAS}

1. Proença RPDC. Alimentação e globalização: algumas reflexões. Ciência e Cultura. 2010;62(4),43-47

2. Dutra de OJE, Marchinni JS. Ciências Nutricionais Aprendendo a aprender. Editora Sarvier; 2008.

3. Campos KR. Movimento slow food: uma crítica ao estilo de vida fast food. [monografia]. Brasília (DF): Universidade de Brasília; 2004.

4. Souza QJOV de, Rodrigues AM. Comportamento de risco para ortorexia nervosa em estudantes de nutrição. Jornal brasileiro de psiquiatria, Rio de Janeiro, 2014 Jul/Sep;63(3), doi:10.1590/00472085000000026

5. American Psychiatry Association. Work Group on Eating Disorders. Practice guidelines for the treatment of patients with eating disorders. 3rd ed. Washington (DC): American Psychiatric Association; 2006.

6. Montagner MA, Pontes JB. Health food junkies: overcoming the obsession with healthful eating. Bratman S. Knight D. New York: Broadway Books; 2000.

7. Brytek-Matera A, Krupa M, Poggiogalle E, Donini LM. Adaptation of the ORTHO-15 test to Polish women and men. Eat Weight Disord. 2014 Jan;19(1):69-76, doi:10.1007/s40519-014-0100-0

8. Bagci BAT, Camur D, Guler C. Prevalence of orthorexia nervosa in resident medical doctors in the faculty of medicine (Ankara, Turkey). Appetit. 2007;49(3):661-666, doi:10.1016/j.appet.2007.04.007

9. Sánchez RM, Moreno AM. Ortorexia y vigorexia: nuevos trastornos de La conduta alimentaria? Transtornos de la conducta alimentaria. 2007;5:457-82

10. Martins MCT, Alvarenga MS, Vargas SVA, Sato KSCJ, Scagliusi FB. Ortorexia nervosa: reflexões sobre um novo conceito. Rev Nutr. 2011;24(2):345-57, doi:10.1590/S1415-52732011000200015

11. Donini LM, Marsili D, Graziani MP, Imbriale M, Cannella C. Orthorexia nervosa: validation of a diagnosis questionnaire. Eat Weight Disord. 2005 Jun;10(2):e28-32

12. Pontes JB, Montagner MÂ. Ortorexia em estudantes de nutrição: a hipercorreção incorporada ao habitus profissional? 2012 —Universidade de Brasília, Brasília, 2012 Dissertação [mestrado] Universidade de Brasília, Faculdade de Ciências da Saúde, Programa de Pós-Graduação em Ciências da Saúde, 2012.

13. Aksoydan E, Camci N. Prevalence of orthorexia nervosa among Turkish performance artists. Eat Weight Disord. 2009 Mar;14(1):33-7, doi:10.1007/BF03327792

14. Nassau BOP. Prevalência de ortorexia nervosa em estudantes de nutrição da universidade católica de Brasília [Monografia]. Brasília, Universidade Católica de Brasília, Curso de Nutrição; 2012.

15. Fidan T, Ertekin V, Isikay S, Kirpinar I. Prevalence of orthorexia among medical students in Erzurum, Turkey. Compr Psychiatry. 2010 Jan/Feb;51(1):49-54, doi:10.1016/j.comppsych.2009.03.001

16. Basaglia P, Freitas ÉA de. Utilização do Questionário de Frequência Alimentar (QFA) para averiguação dos hábitos e preferências alimentares de funcionários públicos da rede estadual de ensino dos municípios de Tuiuti - SP e Morungaba - SP. Saúde em foco. 2015;(7):139-150

17. Levy RB, Claro RM, Bandoni DH, Mondini L, Monteiro CA. Disponibilidade de "açúcares de adição" no Brasil: distribuição, fontes alimentares e tendência temporal. Rev Bras Epidemiol. 2012;15(1):3-12.

18. Brasil. Ministério da Saúde, Secretaria de Atenção à Saúde. Coordenação-Geral da Política de Alimentação e Nutrição. Guia alimentar para a população brasileira. Brasília: Ministério da Saúde; 2014.

19. Leite ACB, Grillo LP, Caleffi F, Mariath AB, Stuker H. Qualidade de vida e condições de saúde de acadêmicos de nutrição. Rev Espaço para a Saúde. 2011;13(1):82-90, doi:http://dx.doi.org/10.22421/1517-7130.2011v13n1p82 
20. Santos AKGV dos, Reis CC, Chaud DMA, Morimoto JM. Qualidade de vida e alimentação de estudantes universitários que moram na região central de São Paulo sem a presença dos pais ou responsáveis. Rev Simbio-Logias. 2014 Dez;7(10)

21. Muniz LC, Zanini RV, Schneider BC, Tassitano RM, Feitosa WMN, González-Chica DA. Prevalência e fatores associados ao consumo de frutas, legumes e verduras entre adolescentes de escolas públicas de Caruaru, PE. Ciênc Saúde Coletiva [online]. 2013 Fev;18(2):393-404, doi:http://dx.doi.org/10.1590/S1413-81232013000200011

22. Bassols AM, Sordi AO, Eizirik CL, Seeger GM, Rodrigues GS, Reche M. A prevalência de estresse em uma amostra de estudantes do curso de medicina da universidade federal do Rio Grande do Sul. Rev HCPA. 2008;28(3):15

23. Freitas AMP, Philippi ST, Ribeiro SML. Listas de alimentos relacionadas ao consumo alimentar de um grupo de idosos: análises e perspectivas. Rev bras epidemiol. 2011 Mar;14(1):161-177 